\title{
Protective effects of Zingiber zerumbet rhizome extract on monosodium urate crystal-induced gout rat model
}

\author{
Anas Nazeih, Asmah Hamid*, Farah Wahida Ibrahim, Nurul Farhana Jufri \\ Faculty of Health Sciences, Universiti Kebangsaan Malaysia, Jalan Raja Muda Abdul Aziz, Kuala Lumpur 50300, Malaysia.
}

\section{ARTICLE INFO \\ Received on: 23/10/2019 \\ Accepted on: 12/02/2020 \\ Available online: 04/04/2020}

\section{Key words:}

Arthritis, gout, inflammation, monosodium urate, Zingiber zerumbet.

\begin{abstract}
Gout is the most common inflammatory arthritis worldwide. Untreated gout causes severe pain and affects the quality of life. Zingiber zerumbet is a well-known traditional plant with anti-inflammatory and antioxidant properties. This study was conducted to evaluate the effects of $Z$. zerumbet extract in gout-induced rats. In this study, 30 male SpragueDawley rats were divided into five groups (six rats in each group): i) normal control group received normal saline and tween 80, ii) model group injected with monosodium urate (MSU) crystal solution, iii) positive control group received $10 \mathrm{mg} / \mathrm{kg}$ celecoxib drug, iv) Z. zerumbet extract at $200 \mathrm{mg} / \mathrm{kg}$, and v) Z. zerumbet extract at $400 \mathrm{mg} / \mathrm{kg}$. The extract was orally administered for 14 days. Rats of all groups (except the normal control group) were injected with MSU crystals $(50 \mu \mathrm{l})$ into the ankle joint on day 11. The ankle joint was measured before injection 10, 24, 48, and 72 hours after injection. On day 14 , all rats were sacrificed. Blood and tissue samples were obtained for the analysis of inflammatory and oxidative stress biomarkers. In this study, the ankle joint diameter rate was significantly reduced by $Z$. zerumbet at 200 and $400 \mathrm{mg} / \mathrm{kg}(p<0.05)$ compared to the model group. The extract prevented inflammation significantly $(p<0.05)$ compared to the model group in white blood cell count, C-reactive protein, and erythrocyte sedimentation rate tests. The two doses of $Z$. zerumbet also significantly $(p<0.05)$ promoted the activity of superoxide dismutase and attenuated 8-isoprostane. Thus, this outcome shows that the administration of $Z$. zerumbet rhizome ethyl acetate extract may be useful and easy to protect against gouty arthritis and the process is probably mediated through its anti-inflammatory and antioxidant properties.
\end{abstract}

\section{INTRODUCTION}

Gout is the most common form of inflammatory arthritis caused by hyperuricemia. It is suffered by a growing number of people, and it has been reported that its incidences have increased over the past 50 years due to certain factors such as changes in lifestyle, increased prevalence of chronic kidney disease, aging of the population, and the use of drugs associated with gout (An et al., 2010). Hyperuricemia is the key precursor of gout development that forms monosodium urate (MSU) crystals. It deposits in joint cavities and soft tissues when the serum uric acid level exceeds the crystal saturation point (Perez-Ruiz et al., 2015). This triggers an acute inflammatory response that usually

\footnotetext{
"Corresponding Author

Asmah Hamid, Faculty of Health Sciences, Universiti Kebangsaan Malaysia, Jalan Raja Muda Abdul Aziz, Kuala Lumpur 50300, Malaysia. E-mail:asmah0901@ukm.edu.my
}

presents with symptoms of excruciating pain and swelling of the joints. In addition, it could lead to a permanent tissue damage which showed as an irreversible impairment of the joint cartilage, and this affects negatively on the life quality of gout patients (Butlin et al., 2012). Several events can set off acute gouty flares, including a high intake of alcohol, high purine food, and sweetened beverage, which can increase the serum uric acid level in the blood (Cronstein and Sunkureddi, 2013).

Allopurinol, corticosteroids, and nonsteroidal antiinflammatory drugs are the main current treatments used to treat gouty arthritis during an acute attack. If it is left untreated at the early stage, tophi formation could be developed over time and surgical intervention would be required (Rasid et al., 2019). Despite the fact that these agents are generally effective in curing gout, the use of these drugs is limited by their aptitude to raise undesirable adverse side effects to gout patients with comorbidities such as cardiovascular, renal, and gastrointestinal diseases (Wallace, 2013). Therefore, alternative therapies are 
needed to provide protective effects to gout patients that could prevent the development of gout disease.

Zingiber zerumbet (L.) Smith is known as "wild ginger" and mainly found in tropical countries. It belongs to Zingiberaceae family which is widely distributed in the Southeast Asia region and has been shown to possess various natural medicinal properties such as anti-inflammatory, anticancer, and antimicrobial (Ruslay et al., 2007; Sulaiman et al., 2010). Previously, the ethyl acetate extract of $Z$. zerumbet has proved to possess a strong antioxidant activity (Ruslay et al., 2007). It has been reported that ethyl acetate extract of $Z$. zerumbet has protective effects against hepatotoxicity in paracetamol-induced rats (Hamid et al., 2011) and against paracetamol-induced nephrotoxicity through its antioxidant activity (Hamid et al., 2012). This extract showed impressive activities in reducing brain cell damage (Hamid et al., 2018a), promoting open wound healing (Hamid et al., 2018b), and protecting red blood cells against oxidative stress (Sam et al., 2019). However, the protective effects of $Z$. zerumbet rhizome ethyl acetate crude extract against gout have never been reported before, and thus, this work is aimed to assess its capability to protect against gout in MSU-induced rat model.

\section{MATERIALS AND METHODS}

\section{Plant material}

Fresh rhizomes of $Z$. zerumbet were purchased from a herbal farm in Temerloh, Pahang, Malaysia, and deposited at Universiti Kebangsaan Malaysia (UKM) Herbarium with voucher specimen number UKMB-29952. The fresh rhizomes were cleaned from soil and dirt. After that, they were washed, chopped into small pieces, and air-dried for 5 days. Then, the rhizomes were grounded into powder form.

\section{Preparation of ethyl acetate extract of Zingiber zerumbet}

The rhizomes powder was soaked in $n$-hexane and ethyl acetate at room temperature for 72 hours, respectively, and the mixture was occasionally shaken from day to day. The mixture was filtered by the Whatman filter paper. The extract was evaporated to dryness at $37^{\circ} \mathrm{C}$ under reduced pressure by a rotary evaporator instrument for maximum elimination of the solvent. Then, the extract was transferred into a clean container and stored at $4{ }^{\circ} \mathrm{C}$. Prior to use, Z. zerumbet ethyl acetate extract was dissolved in tween 80 and suspended in distilled water.

\section{Preparation of MSU crystals}

MSU crystals were prepared as reported previously (Ortiz-Bravo et al., 1993). A mixture of $1 \mathrm{~g}$ uric acid (Sigma, USA), $6 \mathrm{ml} 1 \mathrm{M} \mathrm{NaOH}$, and $194 \mathrm{ml}$ distilled water was heated at $60{ }^{\circ} \mathrm{C}$. After that, the $\mathrm{pH}$ of the mixture was measured (10.5), so it was adjusted and neutralized by adding drops of $\mathrm{HCl}$ solution to make the $\mathrm{pH}$ between 7.1 and 7.2. The solution was cooled slowly by stirring overnight at room temperature. The whitecolored precipitate was collected by centrifuge process at $3000 \times$ $\mathrm{g}$ for 2 minutes and stored at $4{ }^{\circ} \mathrm{C}$ until used. Just before injection into rat ankle joint cavities, the MSU crystals were washed, dried, and suspended in a sterilized phosphate-buffered saline (PBS). The length of the needle-shaped crystals was checked under a polarizing light microscope to be at $12 \mathrm{~mm} \pm 2 \mathrm{~mm}$. About $3 \mathrm{mg}$ of MSU crystals were dissolved in normal saline solution (100 $\mathrm{mg} / \mathrm{ml}$ ) before injecting it into the rats' ankle joint cavity 1 hour before the daily extract treatment.

\section{Experimental animals}

Eight-week-old male Sprague-Dawley rats were obtained from UKM animal house. The rats were kept in a controlled room temperature $\left(25^{\circ} \mathrm{C} \pm 1{ }^{\circ} \mathrm{C}\right)$ with a 12-hours lightdark cycle. Food and water were available ad libitum, and all animal experiments were strictly performed according to the UKM Animal Ethical Committee with an approval number: FSK/2017/ ASMAH/24-MAY/851-MAY-2017-DEC.-2017.

\section{Experimental design}

The rats were randomly divided into five groups: i) control group was administrated orally with tween 80 and distilled water, ii) model group was injected with $50 \mu \mathrm{l}$ MSU solution, iii) positive control group that administered with $10 \mathrm{mg} / \mathrm{kg}$ celecoxib drug, iv) $200 \mathrm{mg} / \mathrm{kg}$ of ethyl acetate extract of $Z$. zerumbet, and v) $400 \mathrm{mg} / \mathrm{kg}$ of ethyl acetate extract of $Z$. zerumbet. For the groups iv and $\mathrm{v}$, the extract was given orally daily for 14 days. On day 11 , all rats (except the control group) were injected with $50 \mu \mathrm{l}$ of MSU crystal solution into the ankle joint cavity, whereas the normal control group was injected with $50 \mu \mathrm{l}$ of normal saline only in their ankle joints.

\section{Joint and swelling rate measurement}

The development of gouty arthritis was assessed by measuring the size of the ankle joint using a micrometer thickness gauge before MSU injection (baseline) and then at 10, 24, 48, and 72 hours after the injection. The joint swelling rate could be calculated as follows:

Joint edema rate $=($ Joint swelling after modeling $/$ Joint swelling before modeling -1$) \times 100$.

\section{Collection of blood and tissue samples}

On day 14, the experimental rats were anesthetized by using KTX (ketamine, xylazine, and zoletil) 0.1 $\mathrm{ml} / 250 \mathrm{~g}$ BW intraperitoneally. Blood samples were taken through cardiac puncture and added into three test tubes: i) Ethylenediaminetetraacetic acid (EDTA) tube for white blood cells (WBCs) count, ii) sodium citrate tubes for the determination of erythrocyte sedimentation rate (ESR), and iii) heparin tube for biochemical assays. The samples in heparin tubes were allowed to clot for approximately 1 hour before centrifugation at 3,000 rpm for 10 minutes before plasma samples were taken and stored at $-40{ }^{\circ} \mathrm{C}$ until use. Finally, the rats were sacrificed humanely by decapitation. Liver tissues were immediately collected, excised, and washed in ice-cold PBS.

\section{Serum C-reactive protein (CRP)}

CRP test was carried out by using a Colorimetric Kit (Elabscience Biotech Co., Ltd., China). Glass test tubes were divided into a blank tube containing $3 \mu \mathrm{L}$ of distilled water, sample tube containing $3 \mu \mathrm{L}$ of serum sample, and standard tube containing $3 \mu \mathrm{L}$ of standard solution. About $0.2 \mathrm{~mol} / \mathrm{L}$ of ammonium chloride buffer and latex particle coated with anti-human CRP antibody were added into each tube. All tubes were mixed and read at 600 $\mathrm{nm}$ before incubated for 5 minutes, and the second absorbance reading was measured again. The level of CRP could be measured as follows: 
CRP concentration $(\mathrm{mg} / \mathrm{L})=\left(\Delta \mathrm{A}_{\text {Sample }}-\Delta \mathrm{A}_{\text {Blank }} / \Delta \mathrm{A}_{\text {Standard }}\right.$
$\left.-\Delta \mathrm{A}_{\text {Blank }}\right) \times$ Concentration of standard $(25.0 \mathrm{mg} / \mathrm{L})$

\section{Erythrocyte sedimentation rate}

The determination of ESR level in blood samples was done according to the method previously described (Bull et al., 1993). Blood was collected in vertical citrate test tubes, mixed, and left vertically for 60 minutes. The length of clear yellowish plasma fluid was measured through a ruler in millimeters per hour $(\mathrm{mm} / \mathrm{hr})$.

\section{Total WBC count}

Total WBCs were counted by following a modified method of Dacie and Lewis (2006). About 2\% of glacial acetic acid was added to the blood samples with the dilution factor of 1:20 to lyse red blood cells. The diluted blood samples were loaded into a hemocytometer slide chamber, and the total WBC was counted under the light microscope.

\section{Serum 8-isoprostane}

Serum 8-isoprostane level was measured by using the enzyme-linked immunosorbent assay (ELISA) kit (Elabscience Biotech Co., Ltd., China) following the manufacturer's protocols. Briefly, biotinylated detection antibody was added into a 96well plate and incubated for 45 minutes at $37^{\circ} \mathrm{C}$. The wells were washed three times, and $100 \mu \mathrm{l}$ of Horseradish peroxidase (HRP) conjugate was added following incubation for 30 minutes at 37 ${ }^{\circ} \mathrm{C}$. After washing five times, the substrate reagent was added and incubated for 15 minutes at $37^{\circ} \mathrm{C}$. A stop solution was added, and the samples were read at $450 \mathrm{~nm}$ through a microplate reader.

\section{Superoxide dismutase (SOD)}

The protein concentration that was required to measure the SOD level in the liver tissue homogenate samples was measured by using Bradford assay method (Bradford, 1976). SOD assay was conducted by following Beyer and Fridovich method (Beyer and Fridovich, 1987). One unit of SOD is evaluated as the amount of enzyme that suppresses $50 \%$ of nitro blue tetrazolium reduction.

\section{Statistical analysis}

All data were expressed as mean \pm standard error of the mean. The statistical analysis for all data was analyzed using a one-way analysis of variance (ANOVA), followed by post hoc Dunnett's test. The data of ankle swelling were analyzed using repeated ANOVA and considered statistically significant at $p<0.05$.

\section{RESULTS}

\section{Gross examination of ankle joint diameter}

A gross examination of the ankle joint changes is shown in Figure 1. The ankle joint of the model group (Fig. 1B) showed noticeable swelling and redness around the ankle joint compared to the normal control group that showed normal joint size and color (Fig. 1A). The positive control group (Fig. 1C), $200 \mathrm{mg} / \mathrm{kg}$ Z. zerumbet extract (Fig. 1D), and $400 \mathrm{mg} / \mathrm{kg}$ (Fig. 1E) Z. zerumbet

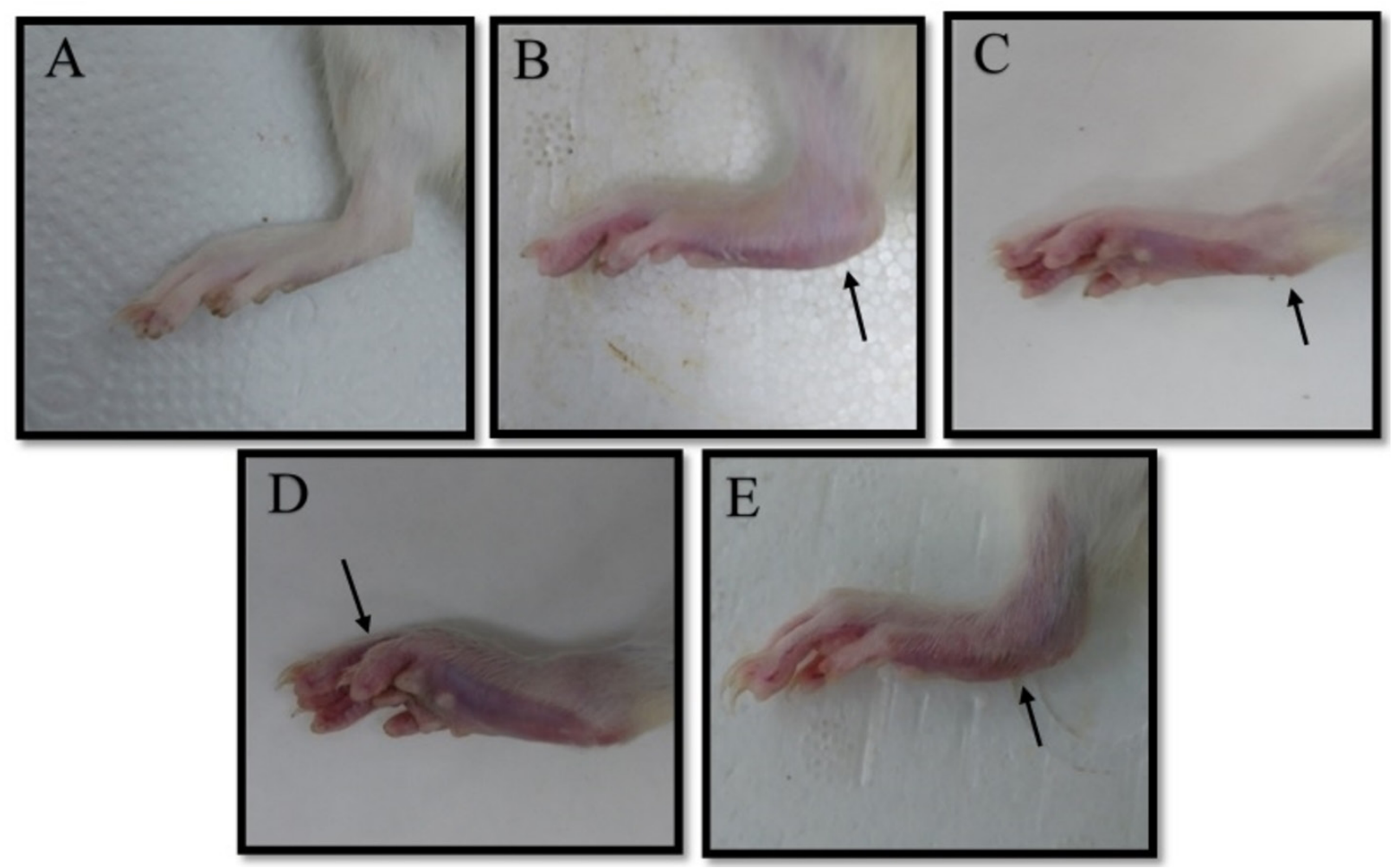

Figure 1. Gross examination of ankle joint in different five experimental groups. (A) Normal control group showed normal ankle joint size and no redness, (B) MSU model group showed redness and joint swelling, (C) positive control group, (D) Z. zerumbet extract at $200 \mathrm{mg} / \mathrm{kg}$ dose, and (E) Z. zerumbet extract at $400 \mathrm{mg} / \mathrm{kg}$ demonstrated a reduction in the joint swelling. Arrow indicates swelling of the ankle joint. 


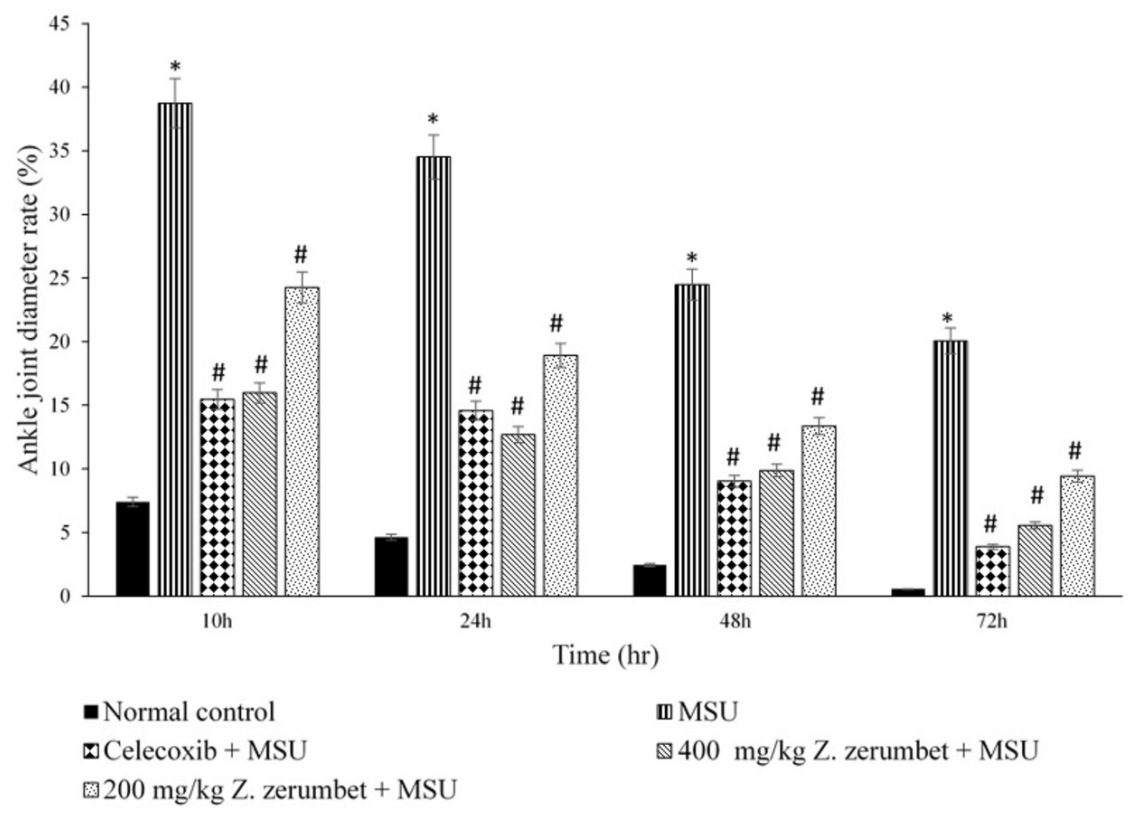

Figure 2. Ankle joint diameter rate of the five different experimental groups. Data are presented as mean \pm standard error mean (SEM) for $n=6$ rats per group. * indicates $p<0.05$ against normal control group and \# indicates $p<0.05$ against MSU group.

extract showed an obvious reduction in swelling and redness of the ankle joint in comparison to the model group.

\section{Ankle joint diameter rate}

As shown in Figure 2, the ankle joint diameter rate of the model group was increased significantly compared to the control group at 10, 24, 48, and 72 hours after the injection of MSU. The joint diameter rate of the positive control group was significantly lower than the model group at 10, 24, 48, and 72 hours after the MSU injection. The Z. zerumbet extract at 200 and $400 \mathrm{mg} / \mathrm{kg}$ doses significantly reduced the diameter rate compared to the MSU model group at 10, 24, 48, and 72 hours.

\section{Inflammatory biomarkers}

Table 1 shows the effects of $Z$. zerumbet extract on inflammatory biomarkers such as WBC count, ESR, and CRP tests. The model group showed a significant increase $(p<0.05)$ in all inflammatory biomarkers compared to the normal control group. The decreased levels $(p<0.05)$ of the inflammatory biomarker levels (compared to the model group) were found in the positive control group and 200 and $400 \mathrm{mg} / \mathrm{kg} \mathrm{Z}$. zerumbet extract groups.

\section{Oxidative stress biomarkers}

Gouty arthritis caused an oxidative stress (Table 1) and showed an increased level of serum 8-isoprostane and decreased endogenous antioxidant SOD activity in the MSU rats' liver compared to the normal control group (Figs. 3 and 4). However, these conditions were oppositely seen in celecoxib and 200 and $400 \mathrm{mg} / \mathrm{kg} \mathrm{Z}$. zerumbet groups compared to the MSU group, as the level of SOD activity was significantly decreased $(p<0.05)$ and significantly attenuated the level of 8 -isoprostane $(p<0.05)$.

\section{DISCUSSION}

Gout is the most common inflammatory arthritis worldwide. It is associated with interactions between MSU crystals and tissues (Tramontini et al., 2004). Uncontrolled and untreated gout patients have an increased risk of developing life-threatening consequences such as cardiovascular diseases, kidney failure, and other comorbidities. Herbal medicine is one of the most popular alternative medicines for inflammatory disorders (Miyata, 2007). In this study, the animal model of acute gout was established by injecting MSU crystals into the ankle joint cavities. To investigate the protective effects of $Z$. zerumbet extract, the rats were given treatment at 200 and $400 \mathrm{mg} / \mathrm{kg}$ for 14 days before inflammatory and oxidative stress tests were carried out.

MSU crystal injection was successfully developed by gout formation in the rats' ankle which was showed by swelling and redness in the ankle joint part in the model group. The model group demonstrated a higher ankle joint diameter rate compared to the normal group resulted from the deposition of MSU crystals and inflammation in the joint tissue as described in a previous study (Sabina et al., 2010). The pretreatment of Z. zerumbet (200 and $400 \mathrm{mg} / \mathrm{kg}$ doses) showed less swelling of ankle joint diameter rats compared to the model group. This suggests that the extract was successfully suppressed the swelling which was stimulated by MSU crystals deposited in the joint cavity, expressing its activity against the development of gouty arthritis. Moreover, Z. zerumbet oral treatment at $400 \mathrm{mg} / \mathrm{kg}$ dose after 10 hours of MSU injection showed the most effective time point and least joint diameter. This may indicate that $Z$. zerumbet crude extract prevented the development of gouty arthritis swelling and implied that it possesses an anti-inflammatory effect, which could provide relief for gouty arthritis.

The presence of MSU crystals in the ankle joint cavity caused an inflammatory response after 72 hours of injection. MSU crystals interacted and caused inflammation in the joint cavity through nucleotide-binding oligomerization domain-like (NOD-like) receptor (NLR) protein inflammasome (Martinon and Tschopp, 2007). In this study, MSU-induced rats in the model group showed remarkably higher levels of CRP, ESR, 
Table 1. Inflammatory biochemical parameters of the different five experimental groups. Data are presented as mean \pm SEM for $n=6$ per group. ${ }^{*} p<0.05$ against normal control and $* *$ indicates $p<0.05$ versus model group.

\begin{tabular}{lccccc}
\hline & Normal control & Model $($ MSU) & Celecoxib + MSU & 200 mg/kg $Z$. zerumbet + MSU & 400 mg/kg Z. zerumbet + MSU \\
\hline ESR $(\mathrm{mm} / \mathrm{h})$ & $2.53 \pm 0.116$ & $10.17^{*} \pm 0.234$ & $2.68^{* *} \pm 0.155$ & $6.23^{* *} \pm 0.285$ & $4.93 * * \pm 0.245$ \\
WBC $(103=\mu \mathrm{L})$ & $8.55 \pm 0.347$ & $14.47^{*} \pm 0.793$ & $9.28^{* *} \pm 0.746$ & $10.57^{* *} \pm 0.255$ & $9.30^{* *} \pm 0.341$ \\
$\mathrm{CRP}(\mathrm{mg} / \mathrm{L})$ & $4.77 \pm 0.251$ & $15.65^{*} \pm 0.411$ & $6.87^{* *} \pm 0.351$ & $12.70^{* *} \pm 0.266$ & $8.87 * * 0.400$ \\
\hline
\end{tabular}

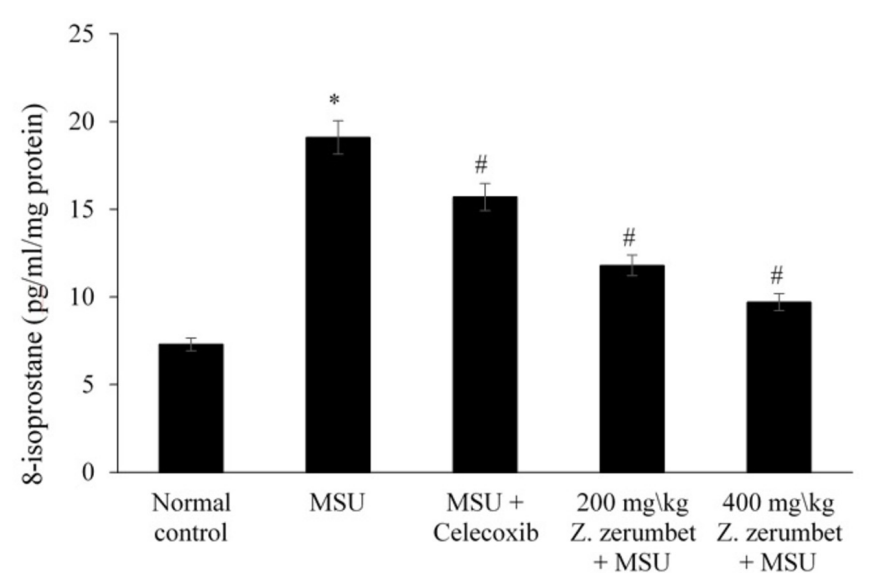

Figure 3. Serum 8-isoprostane levels of the five different experimental groups. Data are presented as mean \pm SEM for $n=6$ rats per group. * indicates a significant difference $(p<0.05)$ compared to the normal control, and \# indicates a significant difference $(p<0.05)$ compared to the model group.

and total WBCs count that have been used to diagnose gouty arthritis compared to the normal control rats. The positive control group and 200 and $400 \mathrm{mg} / \mathrm{kg}$ doses of $Z$. zerumbet extract group showed a significant reduction in the inflammatory biomarkers compared to the model group. This finding is in parallel with a previous study, as the active compound of $Z$. zerumbet extract (zerumbone) successfully inhibited inflammation in rats (Somchit et al., 2012).

Hyperuricemia is one of the etiologies of gout development. In the liver, xanthine oxidase enzyme plays an important role to induce two main reactions such as to produce uric acid in vivo and conversion of hypoxanthine to xanthine and finally produce uric acid (Hille, 2005). Uric acid production can overproduce the reactive oxygen and induce the overproduction of isoprostane. This will cause oxidative injury in vivo and stimulate SOD to inhibit the production of reactive oxygen species (Singh et al., 2017).Z. zerumbet suppressed the production of 8-isoprostane and promoted the activity of SOD in rats' liver in these findings compared to the model group in Figures 3 and 4. This extract induces total antioxidant activities, attenuates destructive oxygen free radicals, and protects against oxidative stress harmful damage (Shaw et al., 2016).

Gout patients and experimental animal model of gout demonstrated a high inflammatory response and elevated oxidative stress (Urano et al., 2002). In this study, Z. zerumbet oral treatment was found to reduce rat's ankle joint swelling, reduce joint inflammation, and attenuate oxidative stress caused by gouty arthritis.

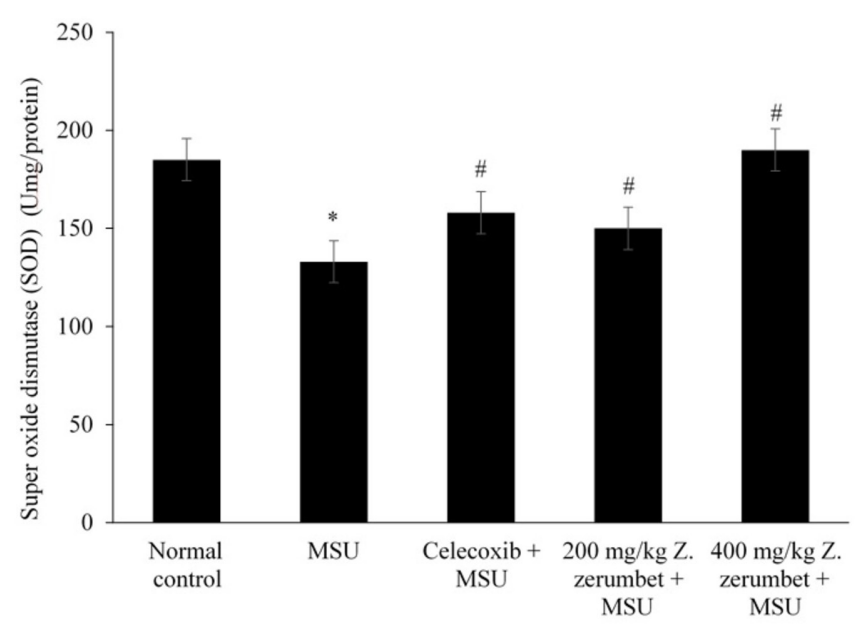

Figure 4. SOD levels in liver tissue homogenate samples of the five different experimental groups. Data are presented as mean \pm SEM for $n=6$ rats per group. * indicates a significant difference $(p<0.05)$ compared to the normal control, and \# indicates a significant difference $(p<0.05)$ compared to the model group (MSU).

\section{CONCLUSION}

Oral administration of $Z$. zerumbet extract effectively protects against gouty arthritis, and the process is mediated through its anti-inflammatory and antioxidant properties. Thus, these findings provide evidence to support the value of $Z$. zerumbet supplementation as an alternative protective treatment against gout.

\section{ACKNOWLEDGMENTS}

This work was supported by Universiti Kebangsaan Malaysia (UKM) under Grant GUP-2016-076.

\section{CONFLICT OF INTEREST}

The author declares no conflict of interest in the data/ research.

\section{REFERENCES}

An J, Yang HJ, Park K, Lee J, Kim BW. Reparatory and preventive effects of oriental herb extract mixture (OHEM) on hyperuricemia and gout, Food Sci Biotechnol, 2010; 19:517-24.

Beyer WF Jr, Fridovich I. Assaying for superoxide dismutase activity: some large consequences of minor changes in conditions, Anal Biochem, 1987; 16:559-66.

Bradford MM. A rapid and sensitive method for the quantitation of microgram quantities of protein utilizing the principle of protein-dye binding. Anal Biochem, 1976; 72:248-54.

Bull BS, Caswell M, Ernst E, Jou JM, Kallner A, Koepke JA, Lewis SM, Lowe GD, Rampling MW, Stuart J. ICSH recommendations 
for measurement of erythrocyte sedimentation rate. J Clin Pathol, 1993; 46:198-203.

Butlin R, Debelle A, Kerth C, Snook RR, Beukeboom LW, Castillo RC, Diao W, Maan ME, Paolucci S, Weissing FJ, Hoikkala A. What do we need to know about speciation? Trends Ecol Evol, 2012; 27:27-39.

Cronstein BN, Sunkureddi P. Mechanistic aspects of inflammation and clinical management of inflammation in acute gouty arthritis. J Clin Rheumatol, 2013; 19:19-29.

Dacie JV, Lewis SM. Practical Hematology. 10th edition, Churchill Living Stone, London, UK, 2006.

Hamid A, Ibrahim FW, Ming TH, Nasrom MN, Eusoff N, Husain K, Latif MA. Zingiber zerumbet L.(Smith) extract alleviates the ethanolinduced brain damage via its antioxidant activity. BMC Complement Altern Med, 2018a; 18:101-7.

Hamid A, Lian CP, Hudin NHS, Ghazali AR, Jufri NF. Zingiber zerumbet rhizomes extract exhibits faster open wound healing in rats. Phcog Res, 2018b; 10:354-60.

Hamid AH, Budin SL, Mohamed RA, Manaf NA, Husain K, Hamid ZA, Mohamed JB, Rahman AH, Collage L. Role of oxidative stress in the protective effects of Zingiber Zerumbet smith ethyl-acetate extract against paracetamol-induced hepatotoxicity in sprague-dawley rats. Aust J Basic \& Appl Sci, 2011; 5:1519-25.

Hamid ZA, Budin SB, Jie NW, Hamid A, Husain K, Mohamed J. Nephroprotective effects of Zingiber zerumbet Smith ethyl acetate extract against paracetamol-induced nephrotoxicity and oxidative stress in rats. J Zhejiang Univ Sci B, 2012; 13:176-85.

Hille R. Molybdenum-containing hydroxylases. Arch Biochem Biophys, 2005; 433:107-16.

Martinon F, Tschopp J. Inflammatory caspases and inflammasomes: master switches of inflammation. Cell Death Differ, 2007; 14:10.

Miyata T. Pharmacological basis of traditional medicines and health supplements as curatives. J Pharmacol Sci, 2007; 103:127-31.

Ortiz-Bravo E, Sieck MS, Ralph Schumacher H Jr. Changes in the proteins coating monosodium urate crystals during active and subsiding inflammation. Immunogold studies of synovial fluid from patients with gout and of fluid obtained using the rat subcutaneous air pouch model. Arthritis Rheum, 1993; 36:1274-85.

Perez-Ruiz F, Marimon E, Chinchilla SP. Hyperuricaemia with deposition: latest evidence and therapeutic approach. Ther Adv Musculoskelet Dis, 2015; 225-33.

Rasid AF, Bajuri MY, Rehir R. Role for surgical excision of gouty tophi in improving hand function. Brunei Int Med J, 2019; 15:9-12.

Ruslay S, Abas F, Shaari K, Zainal Z, Sirat H, Israf DA, Lajis $\mathrm{NH}$. Characterization of the components present in the active fractions of health gingers (Curcuma xanthorrhiza and Zingiber zerumbet) by HPLCDAD-ESIMS. J food Chem, 2007; 104:1183-91.
Sabina EP, Rasool M, Mathew L, EzilRani P, Indu H. 6-Shogaol inhibits monosodium urate crystal-induced inflammation-an in vivo and in vitro study. Food Chem Toxicol, 2010; 48:229-35.

Sam MF, Hamid A, Ghazali AR, Louis SR, Budin SB. Protective effects of Zingiber zerumbet ethyl acetate extract on hydrogen peroxideinduced damage of red blood cells. Sains Malaysiana, 2019; 48:781-90.

Shaw PX, Stiles T, Douglas C, Ho D, Fan W, Du H, Xiao X Oxidative stress, innate immunity, and age-related macular degeneration. AIMS Mol Sci, 2016; 3:196-221.

Singh D, Kumar V, Singh C. IFN- $\gamma$ regulates xanthine oxidasemediated iNOS-independent oxidative stress in maneb-and paraquat-treated rat polymorphonuclear leukocytes. Mol Cell Biochem, 2017; 427:133-43.

Somchit MN, Mak JH, Bustamam AA, Zuraini A, Arifah AK, Adam Y, Zakaria ZA. Zerumbone isolated from Zingiber zerumbet inhibits inflammation and pain in rats. J Med Plant Res, 2012; 6:177-80.

Sulaiman MR, Perimal EK, Akhtar MN, Mohamad AS, Khalid MH, Tasrip NA, Mokhtar F, Zakaria ZA, Lajis NH, Israf DA. Antiinflammatory effect of zerumbone on acute and chronic inflammation models in mice. Fitoterapia, 2010; 81:855-8.

Tramontini N, Huber C, Liu-Bryan R, Terkeltaub RA, Kilgore KS. Central role of complement membrane attack complex in monosodium urate crystal-induced neutrophilic rabbit knee synovitis. Arthritis Rheum, 2004; 50:2633-9.

Urano W, Yamanaka H, Tsutani H, Nakajima H, Matsuda Y, Taniguchi A, Hara M, Kamatani N. The inflammatory process in the mechanism of decreased serum uric acid concentrations during acute gouty arthritis. J Rheumatol, 2002; 29:1950-3.

Wallace JL. Mechanisms, prevention and clinical implications of nonsteroidal anti-inflammatory drug-enteropathy. World J Gastroenterol, 2013; 19:1861-76.

How to cite this article:

Nazeih A, Hamid A, Ibrahim FW, Jufri NF. Protective effects of Zingiber zerumbet rhizome extract on monosodium urate crystal-induced gout rat model. J Appl Pharm Sci, 2020; 10(04):069-074. 